\title{
Mortalidad materna institucional en Caldas 1990 - 1994
}

\author{
Jorge Eduardo Vélez Arango*; Liliana Dávila Arias**
}

\begin{abstract}
RESUMEN: La presente investigación analiza las muertes maternas institucionales que se presentaron en el Departamento de Caldas entre enero $1^{\circ}$ de 1990 y diciembre 31 de 1994 . Se registraron durante dicho período un total de 62 muertes, con un porcentaje de muerte por causa directa del $82,26 \%$ y de causa indirecta del 17,74\%. La tasa de mortalidad obtenida es del 7,27 x 10.000 nacidos vivos, superior a la reportada por países desarrollados. La edad promedio de las pacientes fue de $27,87 \pm 7,63$ años, con un promedio de escolaridad de 5,26 \pm 7,63 años. Se demuestran además bajas frecuencias de control prenatal y altos porcentajes de inestabilidad marital. Los trastornos hipertensivos del embarazo son la primera causa de muerte materna directa, seguidos por la infección y los transtornos hemorrágicos de la gestación.
\end{abstract}

PALABRAS CLAVE: mortalidad materna, tasas de mortalidad materna, prevención de la mortalidad materna.

SUMMARY: This study analyses the institutional maternal deaths which ocurred at the Department of Caldas (Colombia, South America), between January the first and 31th of December of 1994. A total number of 62 deaths were registred during this period, with a porcentaje of direct cause of $82,26 \%$ and indirect of $17,74 \%$. The mortality rate was $7,27 \times 10.000$ viable born which is superior to the registred by developed countries. The mean age of patients was $27,87 \pm 7,63$ years, with a schooling mean of $5,26 \pm 7,63$ years. Low frequencies of prenatal control and high percentajes of marital inestability were demostred. The hipertensive disorders of pregnancy were the first cause of direct maternal death, followed by the infection and the hemorragic disorders of the gestation.

KEY WORDS: maternal mortality, rates of maternal mortality, prevention of maternal mortality.

\section{Introducción}

"No tiene tu muerte excusa. Nunca más será tu hogar lo que fue porque te has llevado un tesoro inefable... el de tu amor infinito, el de tu amor de madre".

Más allá de las definiciones y de las tasas, la muerte materna es el peldaño final de un sendero de desaciertos. Todos los elementos negativos son sus aliados: pobreza, ignorancia, negligencia, oferta inadecuada de servicios de salud, dificultades de acceso a los mismos, carencia de recursos humanos y tecnológicos y hasta la mala suerte de no habitar en países desarrollados. La mortalidad materna es entre nosotros, un problema crítico de salud. Las posibilidades de morir como consecuencia de complicaciones del embarazo, parto y puerperio, son 100 veces superiores para las mujeres de países subdesarrollados que para las de los desarrollados (7-8, 18, 23-24, 27). Paradójicamente las tasas de muerte materna correspondientes a las clases sociales privilegiadas de nuestros países en desarrollo son comparables a las de países desarrollados, diferencias que parecen mostrar el hecho de que disponemos de los conocimientos y recursos necesarios para lograr el parto y el embarazo seguros para

\footnotetext{
* Médico Gineco-obstetra. Jefe Sección Obstétrica. Profesor Asistente Facultad de Medicina. Universidad de Caldas.

Residente III de Ginecología y Obstetricia. Facultad de Medicina, Universidad de Caldas.
}

algunas de nuestras mujeres, pero lamentablemente no para todas. La tragedia de la mortalidad materna debe señalarse como un problema que justifica todos los esfuerzos dirigidos a su prevención. La Organización Mundial de la Salud estima que unas 500.000 mujeres mueren al año, en todo el mundo; como resultado de complicaciones del embarazo y parto, la mayoría de las cuales son prevenibles, considerándose que el 99\% de ellas ocurren en los países subdesarrollados $(18,24,27$, 42). De acuerdo con la OMS, las tasas de mortalidad materna por 10.000 nacidos vivos son de aproximadamente 64 para Africa, 42 para Asia y 27 para América Latina, con un promedio de 3 para el mundo desarrollado en su totalidad (18).

Sin lugar a dudas se ha registrado en las últimas décadas una tendencia a la disminución de las tasas tanto en los países desarrollados como subdesarrollados, demostrándose reducciones de las tasas hasta del $70 \%$. Algunos países han alcanzado tasas entre el 0,4 y $0,5 \mathrm{x}$ 10.000 , como por ejemplo Irlanda, Escandinavia y Suiza $(8,18,39)$. La literatura nacional también permite comprobar esta tendencia a la reducción de las tasas (1-3, 5, $9,12-13)$. Klevens y colaboradores señalan tasas de muerte materna para el Instituto Materno Infantil de Santafé de Bogotá de 109 x 10.000 para el período de 1950 a 1951 y de 26 x 10.000 para 1980 . El municipio de Manizales ha presentado en el último decenio tasas de mortalidad materna entre el 12 y 18 x 10.000 , las cuales continúan siendo preocupantes (11). 


\section{Material y métodos}

La presente investigación puede considerarse de tipo prospectivo descriptivo. Se incluyeron en el estudio todos los casos de muerte materna ocurridos en las instituciones hospitalarias del Departamento de Caldas entre enero $1^{\circ}$ de 1990 y diciembre 31 de 1994 . Se empleó como formulario para recolección de la información el modelo de "HISTORIA CLINICA PERINATAL", diseñado por la Red Perinatal de Caldas y vigente en todo el Departamento. Con el objeto de obtener la información necesaria se instruyó a los directores de todas las unidades de atención médica del Departamento, solicitándoles remitir a la dirección seccional de Salud todos los casos de muerte materna registrados dentro de sus instituciones, con la correspondiente historia perinatal simplificada. Los casos de muerte ocurridos en las instituciones de tercer nivel del Departamento (Hospital de Caldas y Clínica de Villapilar del Instituto de los Seguros Sociales, de Manizales) fueron recolectados por los autores.

Para el manejo de la información se empleó el programa epidemiológico Epi Info, versión 5.01B, utilizando un computador personal IBM compatible.

\section{Resultados}

Durante el período comprendido entre enero $1^{\mathrm{o}}$ de 1990 y diciembre 31 de 1994, se registraron 62 casos de muerte materna institucional en el Departamento de Caldas (51 casos de muerte materna directa $(82,25 \%), 11$ casos de muerte materna indirecta $(17,75 \%))$. De ellas, $53(85,48 \%)$ ocurrieron en las dos instituciones del nivel terciario de la ciudad de Manizales y $9(14,51 \%)$ se presentaron en instituciones de salud de municipios diferentes a Manizales.

La edad promedio de las pacientes fue de $27,87 \pm 7,63$ años. Figura 1. Con relación a la procedencia, 38 de las pacientes $(61,3 \%)$ provenían del área urbana de los municipios. Se halló en el grupo una frecuencia de analfabetismo del $11,4 \%$, con una escolaridad promedio de 5,26 $\pm 3,96$ años. Figura 2. El análisis del estado civil demostró que el 43,9\% de las pacientes carecían de compañero estable. En lo referente a la historia obstétrica

Figura 1

GRUPOS ETARIOS

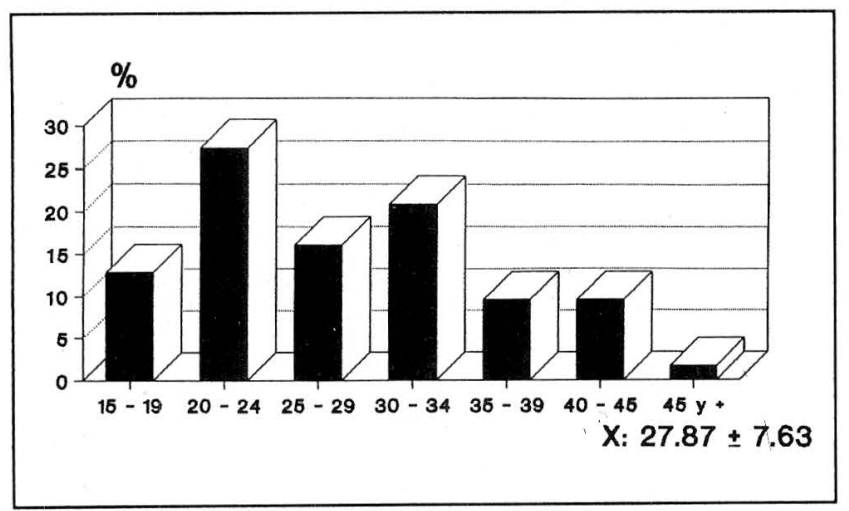

Figura 2

\section{ESCOLARIDAD}

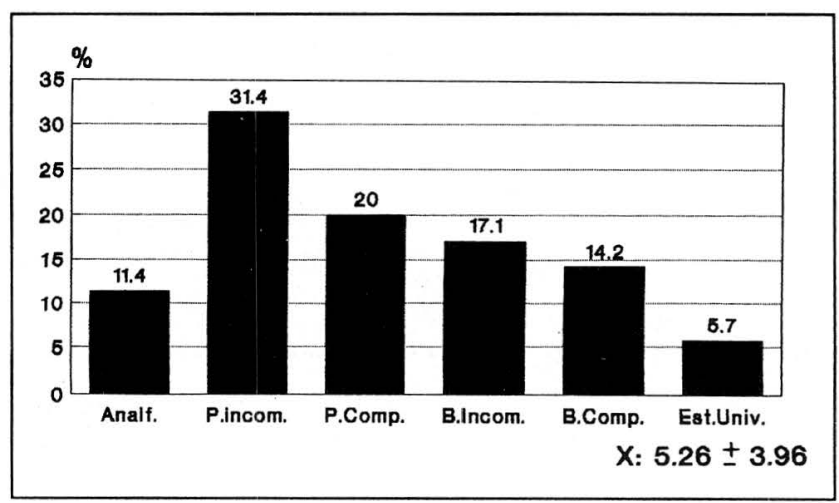

de las pacientes el $27,6 \%$ de ellas eran primigestantes, con un promedio de embarazo de $2,79 \pm 3,51$ y un promedio de hijos vivos al ocurrir la muerte de la madre de 2,07 $\pm 2,53$. Dentro de los antecedentes obstétricos negativos se identificaron frecuencias de mortinato previo en el $8,6 \%$, historia de 1 o más abortos en $17,2 \%$ y de parto pretérmino en el $8,6 \%$ de las pacientes.

El 51,9\% de las pacientes no tuvieron ningún tipo de control prenatal; con un promedio de controles prenatales en el grupo de pacientes que lo tuvieron de sólo 2,12 \pm 2,65 controles. El promedio de edad gestacional al momento de la muerte fue de 30,19 $\pm 8,96$ semanas. La Figura 3 permite observar la clasificación de las pacientes de acuerdo con la edad gestacional al momento de la muerte. Con respecto a la forma de terminación del embarazo, 44 de las pacientes tuvieron parto: 22 de ellas mediante cesárea y las 22 restantes mediante parto vaginal (16 partos normales, 4 partos instrumentados y un parto vaginal asistido en pelvis). Figura 4 . La tabla 1 permite apreciar la forma de terminación del embarazo de todas las pacientes. Cuando se analizó el tipo de persona que atendió el parto, la atención se realizó así: GinecoObstetra: 17 casos, Residente de Gineco-Obstetricia: 16 casos, Médico general: 11 casos.
Figura 3

\section{EDAD GESTACIONAL}

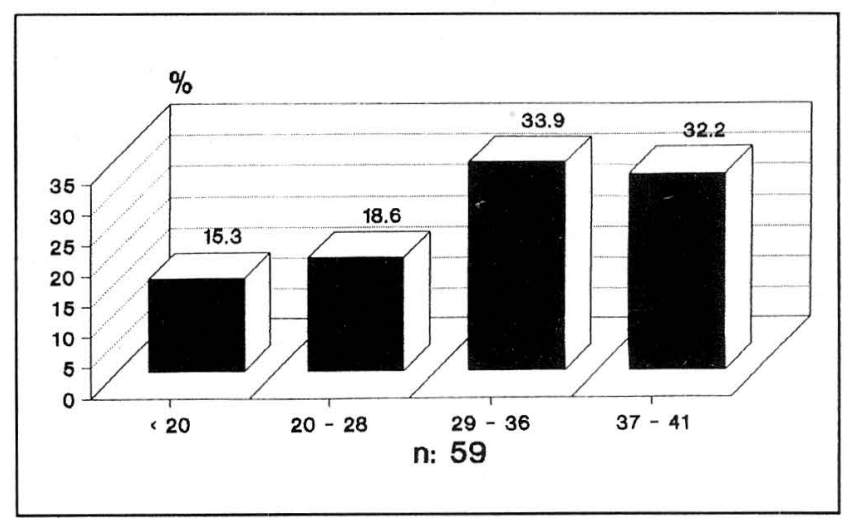


Figura 4

TERMINACION DEL EMBARAZO

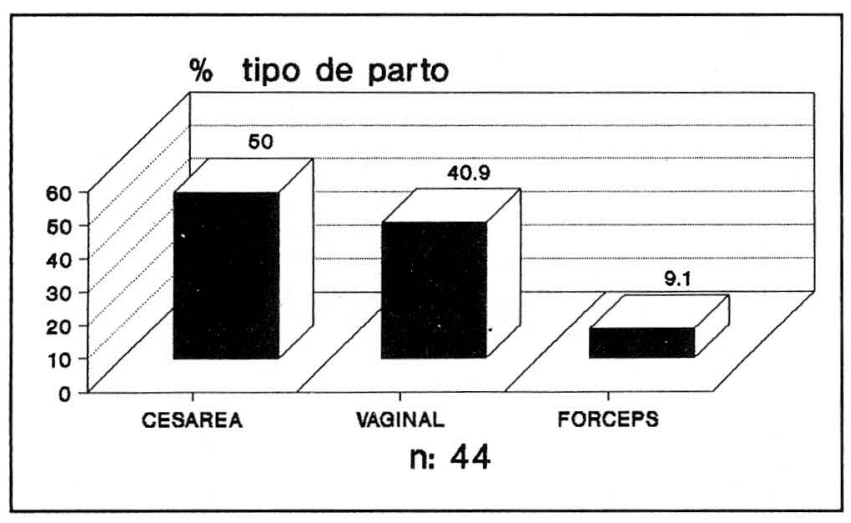

Se presentaron 30 muertes perinatales en el grupo ( 25 antepartos y 5 neonatales precoces). El dato de peso al nacer se obtuvo en 29 casos, hallándose un peso promedio de $2.380 \pm 866 \mathrm{gr}$.

Tabla 1

TERMINACION DEL EMBARAZO

\begin{tabular}{|l|l|}
\hline Terminación del embarazo & Pacientes \\
\hline Cesárea & 22 \\
Parto vaginal & 16 \\
Normal & 4 \\
Fórceps & 2 \\
Asistido en pelvis & 7 \\
Aborto provocado & 9 \\
Muerte materna con feto in útero & \\
Otros: & 1 \\
Mola hidatidiforme & 1 \\
Embarazo abdominal & 62 \\
Total & \\
\hline
\end{tabular}

Dentro de las causas de muerte materna directa, los trastornos hipertensivos de la gestación representan la primera causa con 23 pacientes $(45,09 \%)$, seguidas por las complicaciones infecciosas del embarazo con 13 casos $(25,49 \%)$, discriminados así: 7 casos de aborto séptico provocado y 6 casos de infección durante el parto y el puerperio. En tercer lugar figuran las complicaciones hemorrágicas del embarazo con 4 casos $(7,84 \%)$. Siguen como causas el hígado graso del embarazo con 3 casos $(5,88 \%)$, y el parto obstruido con 2 casos $(3,92 \%)$. Los otros 6 casos $(11,76 \%)$ de muerte materna directa tuvieron como etiología: ruptura uterina: 3 casos, embarazo abdominal: 1 caso, mola hidatidiforme: 1 caso, embolia de líquido amniótico: 1 caso. Figura 5. La Figura 6 permite apreciar las causas de muerte materna indirecta, con las cardiopatías congénitas ocupando el primer lugar con dos casos.
Figura 5

\section{CAUSAS DIRECTAS \%}

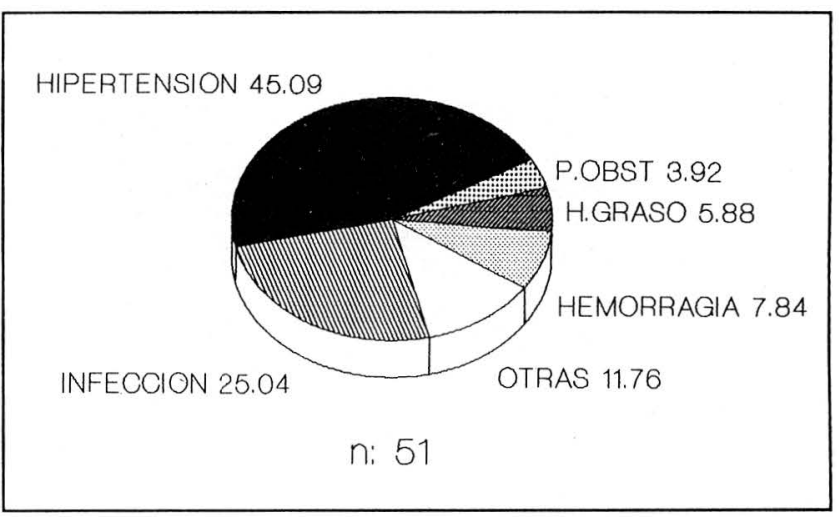

Figura 6

\section{CAUSAS INDIRECTAS \%}

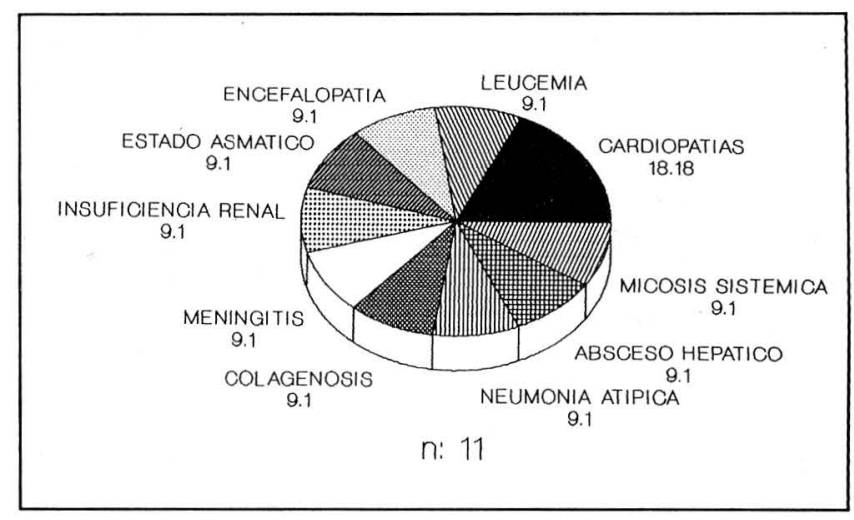

Desafortunadamente y como ocurre con otros informes nacionales, solamente se efectuaron 6 autopsias, lo que representa una frecuencia de necropsias del 9,7\%. En algunas pacientes con proceso infeccioso se realizó anexohisterectomía (5 casos), lográndose conclusiones diagnósticas a partir del informe del espécimen quirúrgico.

Cuando se analizó cada uno de los casos de muerte materna, evaluando la posibilidad de prevención, se consideraron evitables 31 de ellas, lo que permite concluir una frecuencia de muerte prevenible del 50\%. Figura 7.

El intervalo ingreso-fallecimiento de las pacientes fue de 6,75 \pm 7,23 días, falleciendo el $32,8 \%$ de ellas dentro de las primeras 24 horas de hospitalización.

Cuando se intenta una mayor discriminación de las causas de muerte, merecen destacarse las siguientes consideraciones para algunas de ellas: se presentaron 23 muertes secundarias a trastornos hipertensivos de la gestación, clasificadas así: eclampsia: 16 casos $(69,56 \%)$, preeclampsia severa: 4 casos $(17,39 \%)$, hipertensión arterial crónica más preeclampsia sobreagregada: 3 casos $(13,04 \%)$. Debe anotarse que en 5 de las pacientes eclámpticas y en 3 de las pacientes con preeclampsia severa se hallaron datos clínicos y de laboratorio sugestivos del Síndrome de Hellp. Una de las pacientes eclámpticas presentó una ruptura hepática. 
Figura 7

MUERTE PREVENIBLE (CAUSAS DIRECTAS)

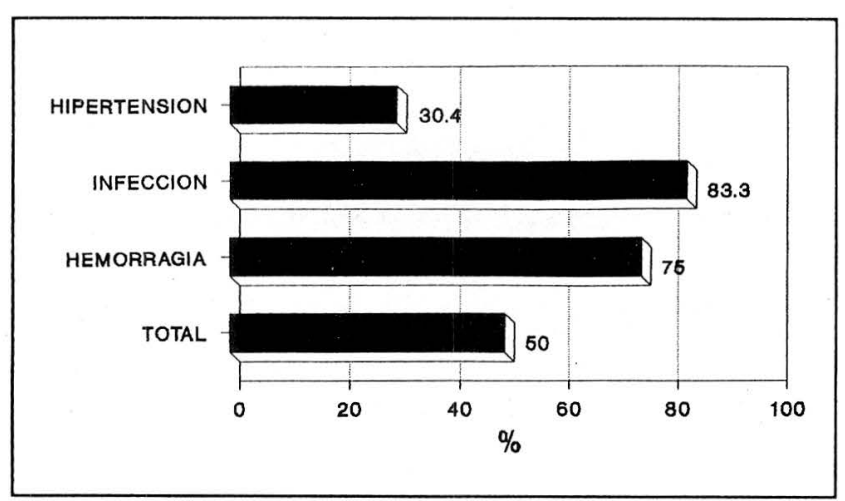

La infección se consideró como causa de muerte en 13 pacientes clasificadas así: aborto provocado: 7 casos; infección obstétrica periparto: 6 casos. Al momento del ingreso, todos los casos de aborto fueron clasificados como aborto séptico grado IV, realizándose anexohisterectomía en 5 de ellas. El $71,4 \%$ de ellas fallecieron dentro de las primeras 24 horas demostrándose con esto la severidad del cuadro. El antecedente de ruptura prematura de membranas se presentó en el 20,3\% de los casos de muerte por infección obstétrica.

Dentro del grupo de pacientes fallecidas como consecuencia de trastornos hemorrágicos $(7,84 \%)$, sólo dos de ellas murieron como resultado directo de la hemorragia; las otras dos pacientes tenían diagnóstico de abruptio placenta y placenta previa, presentando además de la hemorragia complicaciones asociadas como edema agudo del pulmón y sepsis.

Durante este período de 5 años, se presentaron 85.233 nacimientos de productos vivos en las instituciones objeto del estudio, lo que permite establecer una tasa de mortalidad materna de 7,27 x 10.000 nacidos vivos. Al comparar las tasas de muerte materna institucionales, se incluyen acá dos centros de atención de tercer nivel (Hospital de Caldas e Instituto de los Seguros Sociales), con usuarias diferentes, ya que las del primero carecen de cobertura en salud, mientras que las del Instituto de los Seguros Sociales tienen dicha protección. Al obtener las tasas de muerte materna para las dos instituciones, la tasa es de 22,15 x 10.000 nacidos vivos para el Hospital de Caldas y de 2,7 x 10.000 para el Instituto de los Seguros Sociales. La Figura 8 permite apreciar la comparación de las tasas de muerte materna para las dos instituciones, el Departamento de Caldas, Colombia, y las tasas informadas por países desarrollados.

\section{Discusión}

Una tasa de mortalidad materna de 7,27 x 10.000 nacidos vivos, aunque inferior a la reportada para el país en el período 1980-1987 de 11 x 10.000 (Fuente: Estado mundial de la infancia 1990. Tabla 7) y a la de $10 \times 10.000$ de 1990. (Fuente: DANE. Situación de Salud de Colombia.
Ministerio de Salud), aún duplica las tasas de mortalidad materna reportadas para los países desarrollados.

Como puede deducirse de los resultados presentados, deben considerarse como evidentes factores de riesgo para la muerte materna, la baja escolaridad, la inestabilidad conyugal y las bajas frecuencias de control prenatal. Una vez más queda plenamente ratificado el aporte que la condición socio-económica desfavorable hace a la mortalidad materna.

Los trastornos hipertensivos de la gestación representan en esta serie la primera causa de muerte, identificándose para este grupo de pacientes una baja frecuencia de controles prenatales $(31,6 \%)$ y un elevado número de casos de eclampsia $(69,56 \%)$, situaciones estas que invalidan la acción del tercer nivel de atención y permiten destacar nuevamente la importancia del control prenatal adecuado, como única estrategia para el diagnóstico temprano de la patología, con la posibilidad de adoptar conductas de manejo que eviten el progreso de la enfermedad a sus formas graves, en las cuales la mortalidad, como lo revela el estudio es realmente elevada. La alta frecuencia de muertes potencialmente prevenibles $(83,3 \%)$, identificada en el grupo de pacientes que fallecieron como resultado de patologías infecciosas se debe a la existencia en esta categoría de 7 pacientes con muerte secundaria a aborto séptico provocado, situación susceptible de controlar con campañas dirigidas a estimular la sexualidad responsable y el uso de métodos de planificación. Una frecuencia relativamente alta de ruptura de membranas $(20,3 \%)$, en las pacientes fallecidas con diagnóstico de infección obstétrica, permite suponer la existencia de fallas en el manejo de tal condición y en el reconocimiento temprano de la corioamnionitis, su principal complicación.

Además de la baja frecuencia de controles prenatales ya señalada, es evidente la ausencia de una consulta médica preconcepcional bien establecida, durante la cual puedan explicarse a las pacientes los riesgos que supone la gestación en la presencia de patologías como la cardiopatía y todas las demás causas de muerte materna indirecta.

\section{Figura 8}

TASAS DE MUERTE POR 10.000 NACIDOS VIVOS

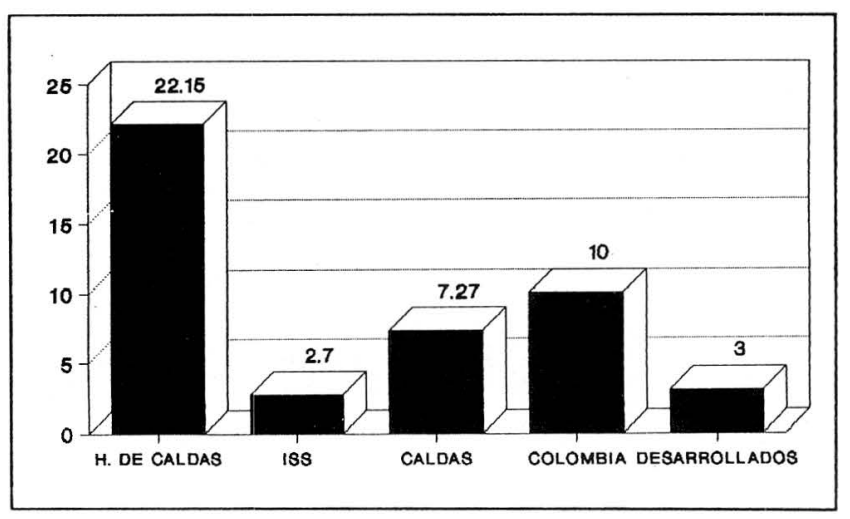




\section{BIBLIOGRAFIA}

1. Alandever GE. Un modelo epidemiológico para el análisis de las políticas de salud. Medellín. Univ. de Antioquia, Facultad de Salud Pública, 1982.

2. Arbeláez N., Patricia y col. Situación de la salud M.I. Boletín Epidemiológico de Antioquia, 555, N 4, oct., nov., dic., 1988.

3. Atención materna y perinatal con criterio de riesgo. República de Colombia, Ministerio de Salud, 1988.

4. Barco V., Virgilio. Desarrollo de una política de salud dentro de una concepción liberal de la economía social. Programa de salud para el período presidencial. Bogotá, 1986.

5. Becerra E. Mortalidad materna en el Instituto Materno Infantil de Bogotá, años 1981-1982. Rev. Col. de Obst. y Ginecol. Vol XXXVII, $\mathrm{N}^{\circ} 5,1986$. p. 328-347.

6. Boletín de la Oficina Sanitaria Panamericana. $2^{\mathrm{a}}$ reunión subregional andina sobre salud M-I. Vol. 107, N 3 , sep. 1989.

7. Boletín médico de I.P.P.F. Los riesgos del embarazo indeseado. Vol. $23, N^{\circ} 1$, feb. 1989. Inglaterra.

- Ayudando a la mujer en todas partes del mundo con planificación familiar y desarrollo. Vol. 23, $\mathrm{N}^{\circ} 2$, abr. 1989. Inglaterra.

- Maternidad sin riesgo en Asia del Sur. Vol. 24, N 3, jun. 1990. Inglaterra.

8. Chelala C. Prevención de la mortalidad materna en las Américas. Perspectivas para los años noventa. Comunicación para la salud $\mathrm{N}^{\circ} 2$. Oficina Sanitaria Panamericana.

9. Díaz Ll., Rodrigo. Mortalidad materna en el H.U.V. Acta médica del Valle, 1979.

10. Dirección general de atención médica M.I. Comité de mortalidad materna. México, 1972.

11. Escobar A., Díaz J. y Posada M. Mortalidad materna en el municipio de Manizales, ene. de 1989-dic de 1989 . Boletín epidemiológico de Caldas, Año $5^{\circ}, \mathrm{N}^{\circ} 1$, ene.-abr., 1991.

12. García O., Guillermo y Náñez, Heliodoro. Mortalidad materna, Hosp. M.I., 1983-1984. Rev. Col. Obst. y Ginec. Vol. 36, N 1, 1986.

13. Gil C., E.; Cataño LO. Mortalidad materna y factores de riesgo. Medellín, Colombia, 1992.

14. JaramilloV., Eduardo. Mortalidad materna Hospital San Jorge, Pereira, 1972-1986. Boletín epidemiológico Pereira. Servicio Seccional Salud Risaralda.

15. Jubiz H., Alfonso y Sánchez M., Carmen. Mortalidad materna comparativa entre 2 períodos: 963-1967 y 1968-1972. Rev. Col. Ginecol. y Obst. Vol 17, $\mathrm{N}^{\circ} 3,1976$. p.: 121-126.

16. Jubiz H., Alfonso y Sánchez M., Carmen. Muertes maternas en el H.U. San Vicente de Paúl. Rev. Col. Obst. y Ginec. Vol. 29, N 4, 1978. p.: 178-182.

17. Klevens BJ. y Díaz R. Tendencias de la mortalidad materna en el Instituto M.I., 1950-1980. Revista Colombiana de Obstetricia y Ginecología, 1986.

18. La prevención de la tragedia de las muertes maternas. Informe sobre la conferencia internacional sobre la maternidad sin riesgo. Nairobi, Kenya. Feb. 1987. O.M.S.
19. Martínez, JW y col. Comportamiento mortalidad materna en el Hospital Universitario San Jorge, 1972-1991. Boletín epidemiológico. Servicio Seccional de Salud del Risaralda.

20. Ministerio de Salud. Programa M.I. Hechos y proyecciones. Bogotá, 1987.

21. Ministerio de Salud. Atención materna y perinatal con criterio de riesgo. Bogotá, 1988.

22. Moreno A. Mortalidad perinatal y materna. Rev. Col. de Obst. y Ginec. Vol. XLI, No 1, 1990. p.: 33-38.

23. Mortalidad materna. Ayudar a las mujeres a evitar el camino de la muerte. Crónica de los OMS, 40, 1986. p.: 195-205.

24. Mortalidad materna en América Latina. Organización Panamericana de la Salud, 1988.

25. Muñoz G., Luis. Mortalidad materna en el Instituto M.I., 1976-1980.

26. Navarro V., Hernando. Aborto séptico. H.U.V. Rev. Col. Obst. y Ginec. Vol. 37, N 1, ene.-feb. 1986.

27. Nubarak, Sussane. Maternidad sin riesgo. Foro mundial de la salud. Revista Internacional de Dsllo Sanitario. OMS. Vol. 10, $\mathrm{N}^{\circ} 2,1989$.

28. OMS. Preparación de indicadores para vigilar los programas realizados en el logro de la salud para todos en el año 2.000, 1981.

29. OMS. Guía para el estudio de la mortalidad materna en los países en desarrollo. Tasas y causas. 1987.

30. OPS/OMS. Evaluación y organización de servicio de salud para atención primaria.

31. OPS. Análisis de la mortalidad. Nuevos usos para indicadores antiguos. Boletín Epidemiológico, Vol. 10, N² 2, 1989.

32. OPS. Mortalidad evitable. ¿Indicador o meta ?. Aplicación en los países en desarrollo. Boletín Epidemiológico, Vol. 11, No 1, 1990.

33. OPS. Manual para el enfoque de riesgo en la atención maternoinfantil. $\mathrm{N}^{\circ} 7,1986$.

34. OPS. Manual para la atención de la madre y el niño. $\mathrm{N}^{\circ} 3,1983$.

35. Rosas R., Laura y otros. Mortalidad materna en el Inst. M.I. Bogotá. Revista Colombiana Obst. y Ginec. Vol. 25, Nº 2, 1974. p. 127-149.

36. Sánchez T., Fernando. Mortalidad materna en el Instituto M.I. Bogotá. Rev. Col. Obst. y Ginec. Vol. 25, N 6, 1974. p.: 395-401.

37. Sánchez T., Fernando. Mortalidad materna, estudio de 761 muertes consecutivas, 1971-1975. Rev. Col. de Obst. y Ginec. Vol 28, Nº 6, 1977. p. 217-229.

38. Sánchez T., Fernando. Factores que intervienen en la atención ginecoobstétrica en América Latina. Rev. Colombiana de Ginecología y Obstetricia. Vol 33, $\mathrm{N}^{\circ}$ 3, 1982.

39. Shanklin DR. y Sheldum S. The pathology of maternal mortality. American Journal of Obstetrics and Gynecology. Vol. 165, $\mathrm{N}^{\mathrm{o}} 4$, 1991. p.: 1126-1155.

40. Texto integrado de ginecología y obstetricia. $3^{a}$ ed. Medellín, 1985.

41. UNICEF. Los niños y el desarrollo en los años 1990. Con motivo de la cumbre mundial en favor de la infancia. UNICEF, 1990.

42. Walker GJ. y otros. La mortalidad materna en Jamaica. Foro mundial de la salud. 1 Vol. 8, 1987. 